\title{
Text messaging's impact on an evidence based medicine tobacco cessation program
}

Steve E. Bronsburg, Nova Southeastern University, College of Osteopathic Medicine's Biomedical Informatics Program, USA, bronsbur@nova.edu

Steven B. Zucker, Nova Southeastern University, College of Osteopathic Medicine's Area Health Education Center, USA, szucker@nova.edu

\begin{abstract}
The leading preventable cause of death in the United States continues to be tobacco use, which is a major global health issue responsible for six million deaths each year with projections by 2030 to exceed eight million. Approximately $90 \%$ of adults own a mobile phone, and over $80 \%$ receive and/or send text messages on a regular basis. Healthcare's acceptance and increased utilization of information technology including mobile phone platforms have expanded research opportunities in the field to not only gather data, but to create it. This randomized trial analyzes the impact of adding motivational interviewing (MI) focused text messages to an evidence based medicine (EBM) tobacco cessation program. Subjects were randomized in the context of a controlled setting into three groups: active; passive; and control. Data was analyzed from the perspective of intent-to-treat. Findings from this randomized trial indicate that MI focused text messages may in combination with an EBM tobacco cessation program positively impact tobacco quit rates.
\end{abstract}

Keywords: Texting, Tobacco, Addiction, Cessation

\section{Introduction}

According to the Centers for Disease Control (CDC, 2014) tobacco addiction is a chronic condition harming nearly every organ of the body causing serious health issues including cancer, heart disease, stroke, and chronic obstructive pulmonary disease a leading cause of death (CDC, 2015; Fiore, et al., 2008). It is also reported by the CDC that approximately 500,000 people die each year from cigarette smoking and over 40,000 deaths each year are attributed to secondhand smoke exposure in the United States. One fifth or $20 \%$ of all deaths in Florida are linked to cigarette smoking (Foster et al., 2012). Approximately 16 million people in the United States suffer from the effects of smoking. In spite of programs developed to provide tobacco prevention services, there still remains a need to address vulnerable populations of all age groups with this health threat (Smart, Richardson \& Bronsburg, 2005). Youth under the age of 18 are vulnerable to tobacco addiction. Each day over 3,000 young people under the age of 18 will have their first cigarette, and over 2,000 of them will move from occasional to daily smokers (CDC, 2016). "If smoking persists at the current rate among youth in this country, 5.6 million of today's Americans younger than 18 years of age are projected to die prematurely from a smoking-related illness (CDC, 2016). This represents about one in every 13 Americans aged 17 years or younger 
who are alive today" (CDC, 2016). The CDC further states that over $18 \%$ of all adults are cigarette smokers, $70 \%$ of them want to quit, and nearly half have attempted to quit during 2010. According to Rollnick and Miller (1995) "motivational interviewing (MI) is a directive, clientcentered counseling style for eliciting behavioral change by helping clients to explore and resolve ambivalence". The goal is to resolve ambivalence to continue the move towards the goal. The MI techniques used for this trial included: normalization, which assures the subject that they like many subjects that feel difficulty with change and affirmations which are supportive statements aimed at increasing a client's confidence. Depending on what group a subject was randomly assigned to in this study, the subject could receive one MI focused text a day and send as many key word texts as they wanted, and in turn receive a MI focused text back.

Evidence based medicine (EBM) evolved from clinical epidemiology and critical appraisal and involves gathering data, information and evidence relevant to answering clinical questions (Sackett, 1997). The addition of MI focused text messages to an EBM tobacco cessation program will provide an additional level of support to individuals trying to quit tobacco. Text will provide ongoing communications assisting overall cessation efforts to finally break the addiction of nicotine. This randomized trial aims to shed light on the impact of receiving a text and using a keyword to receive additional support while attempting to quit tobacco.

\section{Barriers to Quitting Tobacco}

Many tobacco users are reluctant to attempt quitting tobacco for various reasons such as access, support services, the time commitment to attend a tobacco cessation class, travel, cost of tobacco cessation counseling services whether in a group setting or an individual encounter, and the cost of the nicotine replacement therapies such as the patch, other pharmacotherapy or some combination of patch, gum, pharmacotherapy or both. A strong barrier continues to deter access to tobacco cessation services, as well as ongoing tobacco cessation support outside the group classes (Gollust, Schroeder, \& Warner, 2008).

Though access and support are strong barriers deterring quitting tobacco use, there are many methods to seek help with this addiction. Treatment methods for quitting tobacco use include: brief clinical interventions; individual face-to-face or telephone counseling, group counseling; behavioral therapies; extended person-to-person counseling; MI counseling; and utilization of information technology such social media, downloadable applications and text messaging, or some combination of the aforementioned (CDC, 2014). Whittaker et al. (2012) concluded from a review of five studies that text messaging works for tobacco cessation, and Free et al., (2011) stated it doubles quit rates at six months.

\section{Text Messaging in Healthcare}

The significant impact of information technology on healthcare is well documented. According to Fox and Duggan (2012) and Pew (2014), over 90\% of adults have a cell phone, 52\% use their cell phone to gather health information, those between the ages of 18-49 were more likely to use their cell phone to gather health information, $67 \%$ of cell phone owners check their phones for messages even if their phones don't ring or vibrate, $80 \%$ of cell phone users send and receive text messages, and approximately $20 \%$ of cell phone users have health related software applications including smoking cessation applications. 
Mobil health technologies, namely short messaging service text messages, allow healthcare professionals to expand their communications abilities increasing access and reach to patients wherever there is cellular or Internet connection. Text messaging is a powerful communication tool being used in the healthcare industry for a variety of support functions, including reminders for appointments and adherence to medications (Smith, 2011; Free et al., 2011; Rodgers et al., 2005; Whittaker et al., 2009).

Study efforts have been conducted in the area of tobacco cessation and the use of information technology such as websites, email, and increasingly the utilization of text messaging, all in an effort to reduce or stop tobacco use. Still, gaps in research remain in the area of efficient use of text messaging in tobacco cessation efforts (Boal et al., 2015). A review of the literature revealed several important works using text messaging in efforts to help subjects quit tobacco. Rogers et al. (2005) conducted a comparative study utilizing mobile technology by incorporating four weeks of text messages to young smokers whose results showed higher numbers of subjects quitting compared to the control group. Similarly, research conducted using automated email messaging as a tool to improve tobacco quit rates was conducted by Lenert et al., (2004) following the principle of intent to treat "30-day intent-to-quit rates were higher in the individually timed education messages". Intent to treat includes all randomized patients regardless if they withdrew or did not adhere to protocol. Subjects were randomized and analyzed with intent to treat in other studies with similar results (Fishler et al., 1990; Kruse et al., 1990). The results from the Lenert, et al. (2004) study are important findings because the results demonstrate that messaging, in this case, in the form of multiple emails, is a powerful tool to help individuals quit tobacco and break the addiction. According to Bock et al., (2004) mobile technology is able to increase access and deliver a variety of health interventions, thus truly allowing entree to these services to the masses. Perhaps one of the better-known studies on tobacco cessation using texts was conducted by Free et al., (2011) whose findings were that texts double quit rates at six months. These earlier studies include technology such as emails and texts, but they did not add these technologies in combination with an EBM tobacco cessation program, and the texts were not crafted using MI. This is a unique combination of tools for tobacco cessation. This randomized trial utilized a two prong approach of MI focus text messages and an EBM tobacco cessation program in a three group controlled setting to determine what effect if any it had on the programs tobacco quit rates.

Literature is available that incorporates text messages to assist healthcare professionals when working with individuals to quit tobacco (Boal et al. 2015; Free et al., 2011; Haug et al. 2009). This studies focus on quit rates using MI focused texts with an EBM tobacco cessation program. A text message is a powerful communication tool that needs to be utilized in a manner that maximizes its use (Boal et al. 2015; Free et al., 2011; Haug et al. 2009). The correct tool can be under or over used and not accomplish the desired end result. It is anticipated that the results of this study brought increased clarity with new information to using text messaging in combination with other tools to combat tobacco addiction. Also, the results of this randomized trial has provided further insight on the effect of MI focused texts on tobacco quit rates, thus, assisting healthcare professionals to make decisions on how to use text messages with their tobacco cessation efforts when treating tobacco addiction. 
The opportunity to connect to addicted tobacco users through MI focused texts outside of the once a week cessation class setting offers an additional level of support. An MI focused text message might make all the difference between quitting or not. A text can also provide an opportunity to reinforce concepts learned in the tobacco cessation class. Support and reinforcement of newly learned information and skills may help a participant through a tough time. Texting is relatively inexpensive offering many advantages compared to traditional methods of tobacco cessation (Blamford \& Borland, 2014).

\section{Tobacco Cessation Program}

This randomized trial was conducted at Nova Southeastern University, Area Health Education Center's Quit Smoking Now (QSN) Program located in Fort Lauderdale Florida. The texting component of the study was contracted out through Memotext, located in Toronto, Canada. Funding was provided through a grant from Nova Southeastern Universities Health Professions Division. The QSN Program is financially supported with state funds through a contract from the Florida Department of Health. QSN meets for approximately one hour a week for six-weeks and is facilitated by a certified tobacco cessation facilitator. Tobacco cessation facilitators are healthcare professional, community health workers or liaisons, or a member of the community who goes through an approved tobacco cessation facilitators training program and passes the statewide exam certifying them as a tobacco cessation facilitator. Tobacco cessation facilitators provide tobacco cessation counseling to individuals and groups. Most tobacco cessation facilitators work in-group settings where they can leverage their time and effort helping those addicted to tobacco.

Tobacco cessation facilitator group meetings are high-quality encounters that are very productive following an EBM tobacco cessation curriculum. Tobacco cessation facilitators spend a limited amount of time with the subjects at the class, approximately one hour (a lot may happen once the tobacco cessation class ends, and the next one begins approximately one week later). The tobacco cessation program strives to reduce the issues of logistics, access, and cost of tobacco cessation services by offering cessation classes in multiple locations with numerous community partners at no cost to the subject but, it does not address support beyond the class setting (Foster et al., 2012). The addition of MI focused texts to an EBM tobacco cessation program adds that missing support by providing ongoing communications beyond the classroom that assist the tobacco user when they are facing mounting pressures of cravings and setbacks.

\section{Methods}

\section{Study Design and Subjects}

Subjects were recruited over a period of nine months, March 2015 to December 2015. Subjects were recruited from the Nova Southeastern University AHEC QSN program in South Florida. Subjects that signed up for the QSN program were eligible to be recruited for the study. Subjects received a verbal and written explanation of the study, they further signed a consent form and given a copy for their own personal use. To be eligible for the text messaging study, subjects needed to sign up for the QSN program, be a self-declared smoker, between the ages of 18 to 75 , owned a mobile phone with the capacity to receive a text, knew how to text, and were intent on 
quitting tobacco. The tobacco cessation facilitators' collected pre-intervention data, of which a portion was used for this study, which included: gender, race, education level, employment, tobacco use, and the Fagerstorm Tolerence Questions responses to: how soon you smoke after you wake up, how many smokes you have per day, and how frequently you smoke. Postintervention data used for the study was collected by the tobacco cessation facilitator and included use of nicotine replacement therapy and relapse rates. At the end of the study selfdeclared quit status was established via a phone call to the subject by the principle investigator of the study.

The target for the sample size was 60, which was based off of a literature review, the project budget and timeline. The study is set up as a randomized trial. Subjects were randomized using Microsoft Excel RANDBETWEEN function. The range was predefined as 1, 2, or 3 (1 active, 2 passive, or 3 control). A number was entered into Excels spreadsheet using the RANDBETWEEN function and a random integer of 1, 2, or 3 was generated. Once subjects were signed up for the study, and their information was added to the text messaging system, they received a text message welcoming them to the study that stated whether or not they would have the ability to use the keyword. No other information was divulged. Figure 2 illustrates the flow of subjects in the study.

This randomized trial was not looking for effect size specifically, thus a statistical power calculation was not necessary. However, to establish precedence for a sample size that felt appropriate for the study, a literature review was conducted on similar studies with the understanding that there would not be a strong match. The following research studies were noteworthy in the literature review supporting the sample size. A study conducted by Bock et al. (2013) had a sample size of 60 for their study using text messages as a smoking cessation intervention. Two other studies utilized sample sizes that were smaller. A study focusing on user preference for text messaging for a smoking cessation service conducted by Bock et al. (2012) utilized a sample size of 21. Another study conducted by Rios-Bedoya and Hay (2013) used 29 adolescents for a text messaging feasibility study involving alcohol use. Haug et al. (2009) researched feasibility and acceptance of text messaging with 174 subjects in three groups. Haug et al. (2009) concluded that indeed text messaging was valuable for youth based tobacco cessation. 


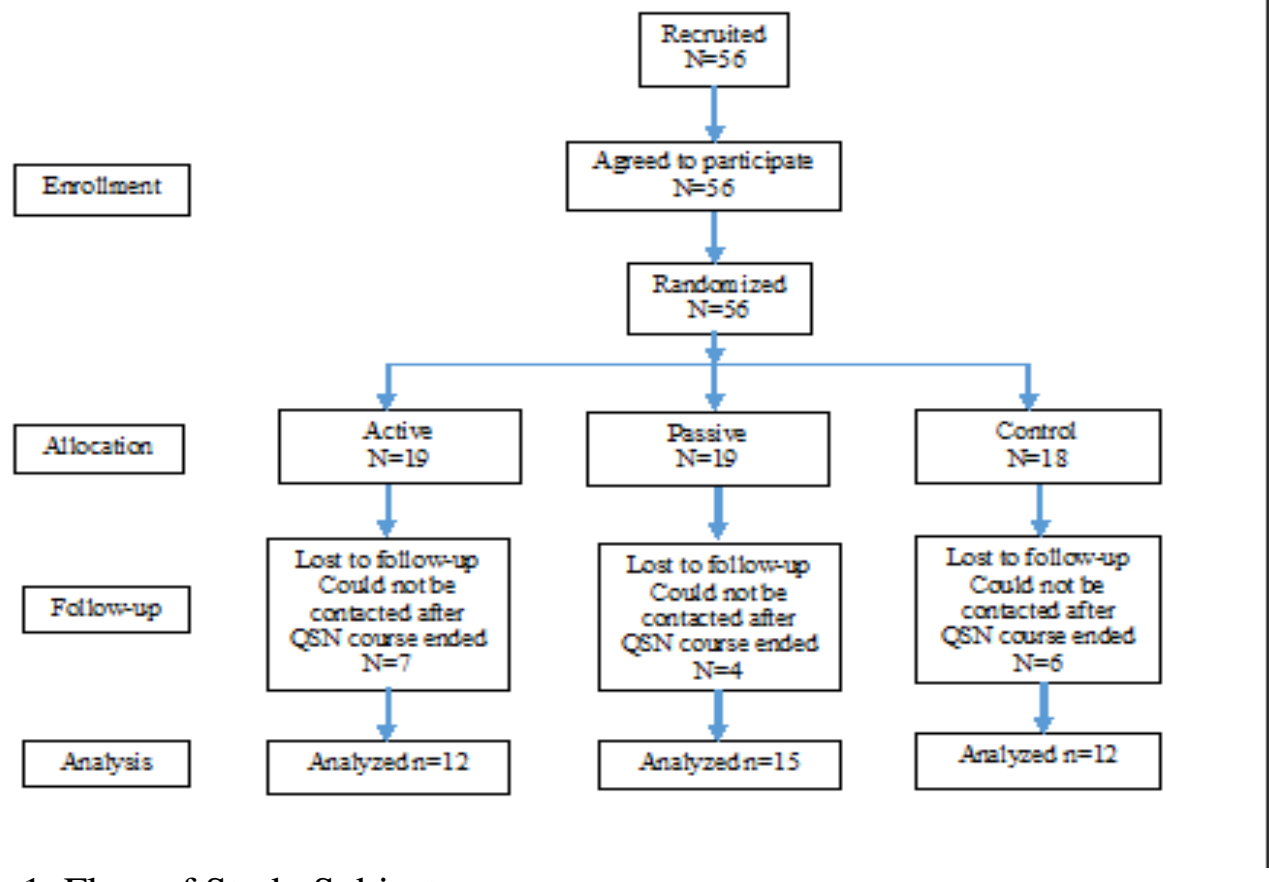

Figure 1. Flow of Study Subjects

\section{Intervention}

A review of the literature on texting and tobacco cessation that yielded several studies of note was a study using three groups (Haug, et al., 2009). The Haug et al., (2009) study focused on feasibility and acceptance of text messaging. The literature review demonstrated that proof of principle has not been done before with three groups regarding the impact of MI focused text messages added to an EBM tobacco cessation program. Subjects for this study using MI focused text messages were randomized into three groups. The smoking cessation intervention for all three groups was the same QSN program that meet once a week for approximately one hour over six weeks. The control group did not receive any text messages throughout the study. The passive group was unidirectional receiving only one MI focused text message daily. The active group was bidirectional receiving one MI focused text message daily and it also had the ability to send a keyword "CRAVE" and receive an MI focused text message. The intention of the keyword was to offer an additional level of support to the subject when they were suffering intense levels of cravings to smoke. The subject types in CRAVE, sends it and receives a text back in a short amount of time. Only the active group had the keyword capabilities for the length of the study. There was a bank of 20 MI focused text messages to respond to the keyword CRAVE available for the active group, and a bank of 25 text messages (daily) for both the active and passive groups.

Figure 1 shows an example of a MI focused text messages that were created by QSN certified tobacco cessation facilitators. 


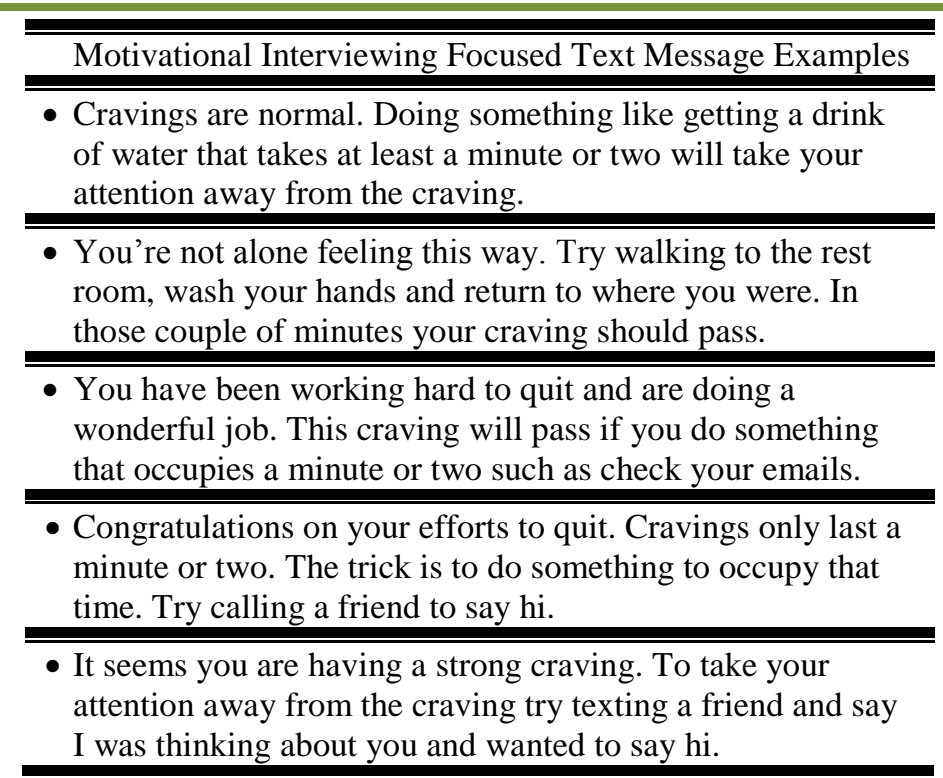

Figure 2. MI Focused Text Message Examples used for both the active and passive groups.

\section{Measures}

\section{Pre and Post Intervention Data}

Per and post-intervention data including the responses to the Fagerstorm questions were collected through a paper questionnaire, and at the end of the study subjects received a telephone call from the principle investigator asking them to self-declare tobacco quit status. During the first QSN meeting all participants who signed up for the class filled out program necessary paperwork which included demographic data used for this study. This data was broken up into two main groups, pre and post-intervention data. The pre-intervention data utilized for this study included gender, race, education level, employment, tobacco use, and a modified Fagerstorm Tolerance Questionnaire. All subjects used nicotine replacement therapy during the study. The type of nicotine replacement therapy was collected by the tobacco cessation facilitator at the end of the tobacco cessation class (six weeks) and recorded. The post-intervention data included quit status and relapse, both gathered from subjects via a phone contact on or after the eighth week of the study.

A review of the literature shows variation on when follow-ups to determine quit status are done on an individuals who attended a tobacco cessation program. Haung et al., 2009 did their followup at the three months while Free et al., 2011 and Boal et al., 2015 did quit rate follow-ups at the six months. There are studies that did follow-ups at seven days (Balmford \& Borland, 2014). Mason et al., 2015 conducted quit rate follow-ups at two months, as did the Florida Area Health Education Center who manages the QSN program (Kerr et al., 2015). Ybarra et al., (2013) did follow-ups at four weeks. The eight week quit status follow-up was based off of the Yabarra et al., (2013) study and the QSN programs existing structure of an eight week quit rate status follow-up. QSN participants which included those volunteering for this study are expected to declare the fourth week of their cessation class as their quit date. The eight week follow-up was 
based on the self-declared quit date of the subject on the fourth week of the cessation classes, and then waiting two additional weeks after the QSN classes had ended to conduct the studies' follow-up. This gave a total of 30 days or one month to follow-up on the subjects quit status, which is comparable to Yabarra et al., (2013) and the QSN programs protocol. Subjects were asked if they used tobacco products over the last 72 hours. This is the same question the QSN program asks. During the follow-up telephone contact, subjects were asked to verify their mailing address so the $\$ 20$ gift card could be mailed to them for participating in the study. All three groups received a follow-up call and were mailed the gift card.

The focus of the study was to look at the impact of MI focused text messages on overall tobacco quit rates while not interrupting the QSN tobacco cessation program. This randomized trial accepted QSN's policy that if a participant finished four weeks of class, they were considered a completer of the program. Nevertheless, Keer et al. (2016) outlined that attending all classes doesn't automatically increase quit rates.

A modified version of the Fagerstrom Tolerance Questionnaire was used to measure nicotine dependency (Fagorstorm, 1978). Addiction level questions included how soon after waking up do you have your first cigarette and the number of cigarettes smoked in a day. Subjects were asked also, to identify if they were daily smokers or not. Responses to the Fagerstorm nicotine addiction questions are indicative to addiction levels (Heatherton, 1991). For example, having a cigarette within 5 minutes after waking indicates a higher level of addiction. Within five minutes is a very high level of addiction. Also, over 11 cigarettes per day indicate a higher level of addiction.

To strengthen the validity of this randomized study, quit rate data was analyzed from the perspective of intent to treat. Meaning, if a participant is lost or missing in a follow-up process, they will still be counted as a smoker and included in the Chi Square analysis. All randomized subjects were part of this analysis. The overall data was analyzed using frequency distributions, and standard deviations and means. Tobacco quit rates were compared using analysis of variance. Intent to treat quit rates were analyzed using Chi-square analysis.

\section{Results}

The flow of the subjects is depicted in Figure 1. A total of 56 participants were enrolled and randomized into the three groups for the study. The subject randomization for the groups were as follows: $33 \%$ in the active group; $33 \%$ in the passive group; and 32\% in the control group. During the follow-up, 17 subjects could not be reached due to disconnected phone numbers or non-responses after a minimum of five attempts. Nevertheless, these subjects were accounted for in intent-to-treat calculations. The final group numbers based off of the 39 subjects were 12 in the active group; 15 in the passive group; and 12 in the control group. 


\begin{tabular}{|c|c|c|c|c|}
\hline \multicolumn{2}{|l|}{ Pre-Interv ention Data } & \multicolumn{3}{|c|}{ Group (Percent) } \\
\hline Variable & & Active & Passive & Coutrol \\
\hline \multirow[t]{2}{*}{ Gervier } & Fenale & $5(417 \%)$ & $8(533 \%)$ & $6(500 \%)$ \\
\hline & Male & $7(583 \%)$ & $7(467 \%)$ & $6(500 \%)$ \\
\hline \multirow[t]{2}{*}{$R x \in$} & White & $3(250 \%)$ & $6(400 \%)$ & $3(25.0 \%)$ \\
\hline & Other & $9(750 \%)$ & $9(600 \%)$ & $9(75.0 \%)$ \\
\hline \multirow[t]{3}{*}{ Eácction Level } & High School $\propto$ less & $4(333 \%)$ & $6(400 \%)$ & $5(417 \%)$ \\
\hline & Some College & $5(417 \%)$ & $6(400 \%)$ & $5(417 \%)$ \\
\hline & Cotlege Grad & $3(250 \%)$ & $3(20.2 \%)$ & $2(166 \%)$ \\
\hline \multirow[t]{4}{*}{ Empioyment } & Full Time & $2(167 \%)$ & $3(200 \%)$ & $5(417 \%)$ \\
\hline & Disabled & $1(83 \%)$ & $6(400 \%)$ & $1(83 \%)$ \\
\hline & Unemployed & $7(583 \%)$ & $3(200 \%)$ & $3(250 \%)$ \\
\hline & Other & $2(167 \%)$ & $3(200 \%)$ & $3(25.0 \%)$ \\
\hline \multirow{2}{*}{ Tobacco Use } & Cigrette & $11(917 \%)$ & $14(93.3 \%)$ & $12(1000 \%)$ \\
\hline & Ciggr & $1(83 \%)$ & $1(67 \%)$ & $0(0 \% \%)$ \\
\hline
\end{tabular}

Table 3. Post-Intervention Data.

\begin{tabular}{|c|c|c|c|c|}
\hline \multicolumn{2}{|c|}{ Post-Interr ention Data } & \multicolumn{3}{|c|}{ Group (Percent) } \\
\hline Variable & & Active & Passive & Coutrol \\
\hline \multirow[t]{3}{*}{$N R T$} & Gun and Patch & $1(83 \%)$ & $5(33.3 \%)$ & $1(83 \%)$ \\
\hline & Lavenges and Patch & $9(75.0 \%)$ & $7(467 \%)$ & $8(667 \%)$ \\
\hline & Other & $2(167 \%)$ & $3(20 \% \%)$ & $3(25.0 \%)$ \\
\hline \multirow[t]{2}{*}{ Quit Stats } & No & $6(500 \%)$ & $9(60,0 \%)$ & $7(58.3 \%)$ \\
\hline & Yes & $6(500 \%)$ & $6(40.0 \%)$ & $5(417 \%)$ \\
\hline \multirow[t]{2}{*}{ Relayse } & No & $6(500 \%)$ & $6(40.0 \%)$ & $5(417 \%)$ \\
\hline & Yes & $6(500 \%)$ & $9(600 \%)$ & $7(58.3 \%)$ \\
\hline
\end{tabular}

Table 2. Fagerstorm Tolerance Responses.

\begin{tabular}{|c|c|c|c|c|}
\hline \multicolumn{2}{|l|}{ Fagerstrom Tolerance Questions } & \multicolumn{3}{|c|}{ Group (Percent) } \\
\hline \multirow{3}{*}{$\begin{array}{l}\text { Variable } \\
\text { Haw soon cffy youwake }\end{array}$} & & Active & Passive & Coutrol \\
\hline & 0-30 Minues & $10(833 \%)$ & $11(73.3 \%)$ & $9(75.0 \%)$ \\
\hline & $31+$ Minutes & $2(167 \%)$ & $4(267 \%)$ & $3(25.0 \%)$ \\
\hline \multirow[t]{3}{*}{ Swoke per Dqy } & $0-10$ & $4(333 \%)$ & $4(267 \%)$ & $5(417 \%)$ \\
\hline & $11-20$ & $5(417 \%)$ & $8(53.3 \%)$ & $3(25.0 \%)$ \\
\hline & $21+$ & $3(25.0 \%)$ & $3(20 \%)$ & $4(33.3 \%)$ \\
\hline \multirow[t]{2}{*}{ Frequency } & Daily & $12(1000 \%)$ & $13(928 \%)$ & $9(75.0 \%)$ \\
\hline & Some Days & $0(00 \%)$ & $1(7.2 \%)$ & $3(25.0 \%)$ \\
\hline
\end{tabular}

The Fisher exact test revealed no difference in Fagerstorm Tolerance Responses by group, $p=0.888$. The Fisher exact test revealed no difference in the amount of smokes-per-day by group, $p=0.682$.

Table 4. Chi Square Analysis

\begin{tabular}{lll}
\hline & \multicolumn{2}{c}{ Count (Percent) } \\
\cline { 2 - 3 } Groups & Quit No & Quit Yes \\
\cline { 2 - 3 } Active & $6(50.0 \%)$ & $6(500 \%)$ \\
Pcessive & $9(60.0 \%)$ & $6(40.0 \%)$ \\
Control & $7(58.3 \%)$ & $5(417 \%)$ \\
\hline \hline
\end{tabular}

Using a chi-square test of independence the percentage of subjects that quit smoking did not differ by group, $\mathrm{c}^{2}(2, N=39)$ $=0.29, p=0.860, \phi_{\text {Cramer }}=0.08$.

Table 5. Chi Square Intent-to-Treat

\begin{tabular}{lcc}
\hline \hline & \multicolumn{2}{c}{ Count (Percent) } \\
\cline { 2 - 3 } Groups & Quit No & Quit Yes \\
\cline { 2 - 3 } Active & $13(68.4 \%)$ & $6(316 \%)$ \\
Parsive & $13(68.4 \%)$ & $6(316 \%)$ \\
Control & $13(68.4 \%)$ & $5(27.8 \%)$ \\
\hline
\end{tabular}

The chi-square test with a Monte Carlo simulation revealed that the percentage of smokers who quit, did not differ by group (2, $\mathrm{N}=56)=0.08, \mathrm{p}=0.959$.

Overall there was no difference between genders with $51 \%$ being male. This was reflected between the groups as well with the control group split at $50 \%$ for both genders. Race was broken out into two categories, white and other. Due to the limited diversity makeup of race of the subjects, the category of other was created as a catch all and consisted of Hispanic, African American and mix. The variable race in the category of other, both active and control groups 
consisted of $75 \%$ other. The variable education level and tobacco use is consistent other findings that state, with higher levels of education individuals are less likely to smoke (Walque, 2007). Unemployed was $58 \%$ for the active group, while the passive group was $20 \%$ and the control group was $25 \%$, yet the active group recorded a higher percentage of quitters $(50 \%)$. Cigarette smokers accounted for $94 \%$ of all subjects, while the remaining 5\% were cigar smokers. See preintervention data in Table 1 for further analysis.

Table 2 shows the results of the modified Fagerstorm responses. Responses to the Fagerstorm question: how soon after you wake up do you smoke, had two categories: 0-30 minutes and 31+ minutes. The 0-31 minute category was high for all three groups: the active group registering $83 \%$, the passive group registered $73 \%$, and the control group registered $75 \%$. Upon awaking from sleep and having a cigarette within 0-30 minutes indicates a strong addiction to nicotine (Fagerstorm, 1978). Smoking 11-21+ cigarettes a day shows a high level of nicotine dependency (Fagerstorm, 1978). Subject responses to the Fagerstorm questions indicated a high level of addiction according to the Fagerstorm scoring system, which tells your level of nicotine addiction, although it's not a diagnosis (Fagerstorm, Heatherton \& Kozlowski, 1992). Nearly all three groups were smoking daily with the active group at $100 \%$ for this question. Also, of note was that $80 \%$ of the subjects were using a combination of nicotine replacement therapy. Combination of nicotine replacement therapy such as the patch and a lozenge or gum will increase quit rates, although the effect of other services or tools including technology need more exploration (Fiore et al., 2008).

Table 3 displays the post-intervention data. The lozenge and patch was the major choice of nicotine replacement therapy for all three groups with $75 \%$ of the active group, $46 \%$ of the passive group and $66 \%$ of the control group choosing this method to assist them quitting tobacco use. Relapse percentages with all three groups were high. The active group relapse was $50 \%$, the passive group was $60 \%$ and the control group was 58\%. The relapse rates for the QSN program were $36 \%$ while the overall results for subjects of this study were $56 \%$. This is $20 \%$ higher than the QSN program. A potential explanation for this larger number may be due to the smaller number of subjects for this study, 39 verses 357 prolonged abstinence used for the QSN program analysis (Kerr et al., 2015).

Table 4 shows the percentage of those that quit tobacco. In the variable race under other, the active group quit rate is $50 \%$, the control group quit rate was $41 \%$ while the passive group quit rate was $40 \%$. The active group quit rate from the study was $13 \%$ higher than the QSN quit rates. The overall quit rates were $43 \%$. Quit rates for the active group, which included getting a text message and having the ability to use a keyword (bidirectional) had the highest quit rates at $50 \%$ followed by passive group (unidirectional) with a quit rate of $40 \%$ and finally the control group at $41 \%$. Having the ability to not only receive an MI supportive text and have use of a keyword seems to have had a positive impact on tobacco quit rates. The QSN program quit rates were $37 \%$ at the 30-day prolonged abstinence (Kerr et al., 2015). The overall results of this study had a quit percentage at the 30 -day prolonged abstinence of $43 \%$, which is $14 \%$ higher than the QSN program at $37 \%$. All three groups recorded higher quit percentages then the QSN program at the 30 -day prolonged abstinence. The active group was $25 \%$ higher, the passive group was $6 \%$ higher and the control group was $8 \%$ higher than the QSN program. This is a notable increase in 
those that quit tobacco use, although other contributing variables such as nicotine replacement therapy, the EBM QSN program, and the small number of subjects must be taken into account. According to the American Cancer Society (2016) four to seven percent of those that try to quit make it without medicine or other help, and $25 \%$ who use medication are successful after six months. Table 5 displays the intent to treat data on quit percentages. The active and passive groups were $31 \%$ and the control group was $27 \%$, both below the QSN program percentages at the 30-day prolonged abstinence.

\section{Discussion}

This study makes a unique contribution in understanding how MI focused text messaging may effect treating nicotine addiction in an EBM tobacco cessation program. This is one of few studies that incorporate MI focused text messages into an EBM tobacco cessation program. There are limited studies incorporating text messaging into a tobacco cessation programs that are focused on what might be the best use of texting in efforts to reduce tobacco use or increase tobacco quit rates (Ybarra et al., 2012). This randomized trial takes a significant step toward identifying an ideal mix of MI focused text messaging to an EBM tobacco cessation program. Findings from this study furthers our understanding of how technology can be used to treat tobacco addiction. Final results of the randomized trial lean towards suggesting that MI focused text messages may very well have had a positive impact on tobacco quit rates when coupled with an EBM tobacco cessation program. Those in the study that had use of a keyword had the highest quit rates of the three groups. Earlier similar studies had mixed outcomes using text messaging for tobacco cessation (Boal et al. 2015; Free et al. 2011). Further research in this area still needs to be conducted to reveal what kind of impact that other variables may have had on increasing tobacco quit rates under similar conditions.

Findings from this randomized trial need to be interpreted in regards to its limitations. There were several strengths and limitations of this randomized trial. Strengths included that it was a randomized trial, the text messages were MI focused, the text messages were added to an EBM tobacco cessation program, and subjects were analyzed per intent-to-treat. Participants were randomized into one of the three groups (active, passive and control). This is a unique study because of the three-group model and MI focused text messages incorporated into the EBM tobacco cessation program. MI focused text messages were developed by QSN tobacco cessation facilitators who have ongoing training in MI and are certified tobacco cessation facilitators. The EBM tobacco cessation program was developed across the state of Florida with several colleges of medicine and is updated yearly. Intent-to-treat analysis was used to calculate all three groups quit rates. Intent to treat captures all of the subjects regardless if they completed the study avoiding findings that may mislead due to a subject dropping out (Lachin, 2000).

The limitations revolved around available limited resources for this randomized trial. The limitations were a small sample size, non-use of a biochemical analysis to test tobacco abstinence, a limited pre and post-intervention data, and the 30-day follow-up. The sample size was limited to 60 subjects based on time and resources allotted for the study. Several studies had utilized similar sized samples with mixed results (Bock et al. 2013; Brock et al., 2012; RiosBedoya \& Hay, 2013). Though there is a notable difference between the groups with this sample size, the difference is not statistically significant. Subjects self-declared quit rates were recorded 
rather than biochemically verified abstinence (SRNC Committee, 2002). Biochemical analysis to verify tobacco abstinence include a carbon monoxide breath test determines how much carbon monoxide in in your lungs, and/or a cotinine test, which test how much nicotine in in your system. Although biochemical analyses are strong indicators of tobacco abstinence, they have their limits (Free et al., 2011). Carbon monoxide levels are good up to 24 hours after tobacco use, and cotinine tests have a half-life of 20 hours. The pre and post-intervention data could be expanded on to include a more insightful picture of the subjects by including data such as what stage of change they are in and prior quit attempts, as well as text message feedback including satisfaction levels with the texts. Resources and time permitting, the length of follow-up on selfdeclared quit rates could have been expanded to three to six months in line with other significant studies (Free et al., 2011; Haug et al., 2009; Mason et al., 2015).

Despite these limitations, this randomized trial offers strong insight into the utilization of MI focused text messages impact on an EBM tobacco cessation programs tobacco quit rates. The quit rate outcome of this randomized trial at the 30-day mark for all three groups (active group $50 \%$, passive group $40 \&$, control group 41\%) were higher than the QSN programs (37\%). The active group was $25 \%$ higher, the passive group was $6 \%$ higher and the control group was $8 \%$ higher than the QSN programs 30-day quit rate. Quit rate outcomes for this randomized trial seem to suggest that MI focused text messages may offer a positive impact on increasing the quit rate when coupled with an EBM tobacco cessation program. Let's not lose sight of the nicotine replacement therapy and the QSN EBM tobacco cessation program that the MI focused text messages augmented in the fight against tobacco addiction. The nicotine replacement therapy and the EMB program are crucial components of a tobacco cessation program, although not the nucleolus of this study.

The results of this randomized trial are exciting. MI focused text messages appear to have a positive effect on tobacco quit rates when added to an EBM tobacco cessation program. More study needs to be done in this area of addiction. It is anticipated that this randomized trial will be repeated by the principle investigator with increased numbers of subjects, and expanded variables to test this study's findings and conclusions for generalizability. The pure number of text messages subjects receive, and the duration of these messages is an area that requires further exploration. Perhaps subjects need more MI focused texts while attempting to quit tobacco and possibly extending this support well past post quit (Herd, Borland \& Hyland, 2009; Segan, 2008). Future research in tobacco cessation should include the use of other technology tools besides text messaging including current technological devices that are readily available such as the Fitbit and Apple's watch.

\section{Acknowledgments}

Thank you to the anonymous referees for their critical review of this paper. The valuable insight reflected in their comments and suggestions were noted, appreciated and incorporated. Also, we want to thank the accepting editors and editorial board of the Online Journal of Applied Knowledge Management for their assessment, feedback and recommendations. Finally, we wish to thank the Knowledge Management (KM) Conference 2016 program committee chairs, cochairs, organizers, coordinators, and the program committee members for all their efforts to provide researchers and scientist an opportunity to showcase their work. 


\section{References}

American Cancer Society (2016). Guide to quitting smoking. American Cancer Society. Retrieved from: http://www.cancer.org/healthy/stayawayfromtobacco/ guidetoquittingsmoking/guide-to-quitting-smoking-success-rates.

Balmford, J., \& Borland, R. (2015). How do smokers use a smoking cessation text messaging intervention? Nicotine and Tobacco Research, 16(12), 1586-1592.

Boal, A., L., Abroms, L., C., Simmens, S., Graham, A., L., \& Carpenter, K., M. (2015). Combined quitline counseling and text messaging for smoking cessation: a quasiexperimental evaluation. Nicotine and Tobacco Research, 1-8.

Bock, B., Graham, A., Sciamanna, C., Krishnamoorthy, J., Whiteley, J., \& Carmona-Barros, R., (2004). Smoking cessation treatment on the Internet: Content, quality, and usability. Nicotine and Tobacco Research, 6, 207-219.

Bock, B., Heron, K., Jennings, E., Magee, J., \& Morrow, K. (2012). User preferences for a text messaging-based smoking cessation intervention. Health Education and Behavior, 40(2), 152-159.

Bock, B., Heron, K., Jennings, E., Morrow, K., Cobb, V., Magee, J.,...Foster, R., (2013). A text delivered smoking cessation intervention: The initial trial of TXT-2-Quit: randomized controlled trial. Journal of Medical Internet Research, 1(2)1-11.

Center for Disease Control and Prevention (2016). Smoking and Tobacco Use: Fast Facts, Disease and Death. Retrieved from:

http://www.cdc.gov/tobacco/data_statistics/fact_sheets/fast_facts/index.htm.

Center for Disease Control and Prevention (2015). Smoking and Tobacco Use: Fast Facts. Retrieved from:

http://www.cdc.gov/tobacco/data_statistics/fact_sheets/fast_facts/index.htm.

Center for Disease Control and Prevention (2014). Smoking and Tobacco Use: Fast Facts. Retrieved from: http://www.cdc.gov/tobacco/data_statistics/fact_sheets/index.htm\#core4.

Center for Disease Control and Prevention (2014). 2014 Surgeon General's Report: The Health Consequences of Smoking-50 Years of Progress. Retrieved from: http://www.cdc.gov/tobacco/data_statistics/fact_sheets/youth_data/tobacco_use/index.ht $\underline{\mathrm{m}}$.

Fagerstorm, K., O., Heatherton, T., E., \& Kozlowski, L., T. (1992). Nicotine addiction and its assessment. Ear Nose and Throat Journal, 69(11), 763-767.

Fagerstorm, K., O. (1978). Measuring degree of physical dependence to tobacco smoking with reference to individualization of treatment. Addiction Behavior, 3(3-4), 235-241.

Fisher, L., D., Dixon, D., O., Herson, J., Frankowski, R., K., Hearron, M., S., \& Peace, K., E., (1990). Intent to treat clinical trials. Statistical Issues in Drug Research and Development. New York, NY: Marcel Dekker.

Fiore, M., C. et al. (2008). Treating Tobacco Use and Dependence: 2008 Update. Clinical Pratcice Guideline. Rockville, MD: US Department of Health and Human Services.

Foster, R., Bronsburg, S., Zucker, S., \& Saldias, G., (2012). Development and Implementation of an Innovative Tobacco Cessation Systems Change Model in Regional Safety-Net Sites in South and Central Florida. American Public Health Association Annual Meeting. 
Fox, S., \& Duggan, M. (2012). Mobile health 2012. A project of the pew research center: mobile health 2012. Pew Research Center's Internet and American Life Project. Washington, D.C.: Pew Research Center's Internet and American Life Project. Retrieved from: http://www.pewinternet.org/Reports/2012/Mobile-Health.aspx.

Free, C., Knight, R., Robertson, S., Whittaker, R., Edwards, P., Zhou, W., Roberts, I., G. (2011). Smoking cessation support delivered via mobile phone text messaging (txt2stop): a single-blind comparative study. The Lancet, 378, 49-55.

Gollust, S. E., Schroeder, S., A., Warner, K. E., (2008). Helping smokers quit: understanding the barriers to utilization of smoking cessation services. The Milbank Quarterly, 86(4), 601627.

Haug, S., Meyer, C., Schorr, G., Bauer, s., \& John, U. (2009). Continuous individual support of smoking cessation using text messaging: A pilot experimental study. Nicotein and Tobacco Research. Doi: 10.1093/nrt/nrt084.

Heatherton, T., F., Kozlowski, L., T., \& Frecker, K., O. (1991). The Fagerstrom Test for Nicotine. British Journal of Addiction, 86, 1119-1127.

Herd, N., Borland, R., \& Hyland, a., (2009). Predictors of smoking replase by duration o abstinence: findings from the international tobacco control (ITC) four country survey. Addiction, 104, 2088-2099. Doi:10.1111/j.1360-0443.209.02732.x

Kerr, A., N., Graalum, E., Rainer, J., Kittelson, V., \& Lien, B. (2015). Florida Area Health Education Centers interventions: FY14 final report. Professional Data Analysts Inc. Minneapolis, MN.

Kruse, R., L., Alpher, B., S., Reust, C., Stevemer, J., J., Shannon, S., \& Williams, R., H., (2002). Intent to treat analysis: who is in? who is out? Journal of Family Practice, 51, 969-971.

Lachin, J., M. (2000). Statistical considerations in the intent-to-treat principle. Controlled clinical Trials, 21, 167-189. doi:10.1016/S0197-2456(00)00046-5.

Lenert, L., Munoz, F., M., Perez, L., E., \& Bansod, A. (2004). Automated email messaging as a tool for improving quit rates in an internet smoking cessation intervention. Journal of the American Medical Informatics, 11(4), 235-240.

Mason, M., J., Mennis, J., Zaharakis, N., M., \& Way, T. (2015). The dynamic role of urban neighborhood effects in a text messaging adolescent smoking intervention. Nicotine and Tobacco Research, 00(00) 1-7.

Pew (2014). Pew internet project: mobile technology face sheet. Retrieved from: http://www.pewinternet.org/fact-sheets/mobile-technology-fact-sheet/.

Rios-Bedoya, C., F. \& Hay, C. (2013). Feasibility of using text messaging for unhealthy behaviors screening in a clinical setting: a case study on adolescent hazardous alcohol use. Journal of the American Medical Informatics Association, 20, 373-376.

Rogers, A., Corbett, T., Bramley, D., Ridell, T., Wills, M., Lin, R., B., \& Jones, M. (2005). Do u smoke after txt? Results of a comparative study of smoking cessation using mobile phone text messaging. Tobacco Control, 14, 255-261.

Rollnick, S., \& Miller, W., R. (1995). What is the motivational interviewing? Behavior and Cognitive Psychotherapy, 23, 325-334.

Sackett, D., L. (1997). Evidence-based medicine. Seminars in Perinatology, 21(1), 3-5.

Segan, C., J., Borland, R., Hanna, a., \& Stillman, S., (2008). The challenge of embracing a smoke-free lifestyle: a neglected area in smoking cessation programs. Health Education Research, 23, 1-9. Doi:10.1093/her/cy1157. 
Smart, A., Richardson, B. \& Bronsburg, S., E. (2005). The Florida AHEC Network as a Vehicle for Tobacco Prevention Education. The National AHEC Bulletin, 22(1), 24-25.

Smith, A. (2011). Americans and text messaging. Washington DC: Pew Internet \& American Life Project. Retrieved from: http://pewinternet.org/Reports/2011/Cell-Phone-Texting2011/Main-Report/How-Americans-Use-Text-Messaging.aspx.

SRNC Committee (2002). Biochemical verification of tobacco use and cessation. Nicotine Tobacco Research, 4(2), 149-159.

Walque, D. (2007). Does education effect smoking behaviors? Evidence using the Vietnam draft as an instrument for college education. Development Research Group, The World Bank, 877-895.

Whittaker, R., Borland, R., Bullen, C., Lin., R., B., McRobbie, H., \& Rodgers, A. (2012). Mobile phone-based interventions for smoking cessation. Cochrane Database of Systematic Reviews. CD006611. doi:10.1002/14651858.CD006611.

Ybarra, M., L., Holtrop, J., S., Bagci Bosi, A., T., \& Emri, S. (2013). Design considerations in developing a text messaging program aimed at smoking cessation. Journal of Medical Internet Research, 14e103 doi10.2196/jmir.2061.

\section{Authors' Biographies}

Steve E. Bronsburg received his Doctorate of Philosophy (Ph.D.) and Master of Science (M.S.) degrees in Information Systems from Nova Southeastern University's (NSU) Graduate School of Computer Information Systems (GSCIS) now renamed the College of Engineering and Computing. He also holds a Master of Health Services Administration (M.H.S.A.) degree from Florida International University (F.I.U.), and is a Certified Tobacco Treatment Specialist (C.T.T.S.) through the University of Medicine and Dentistry in New Jersey (UMDNJ). Dr. Bronsburg is an Assistant Professor at NSU in the College of Osteopathic Medicine's (COM's) Biomedical Informatics Program. Prior to his faculty appointment, Dr. Bronsburg served as the Assistant Director of the state funded Tobacco Training and Cessation Program at the NSU COM's Area Health Education Center (AHEC). Dr. Bronsburg authored and co-authored several presentations and articles on the AHEC Tobacco Program at the Annual Meetings of the American Public Health Association and for the National AHEC Organization. In addition, Dr. Bronsburg has worked on several grant funded projects including avatar training modules for healthcare providers and developed a smartphone application to help address individuals working on reducing their alcohol use. He has just completed a research project focused on a text reminder and support system for tobacco cessation and has written about the role of information technology project management in healthcare. Dr. Bronsburg has co-authored articles on the NSU COM Biomedical Informatics Program, presented on HIPAA and Meaningful Use, and continues to be involved in healthcare technology related research. Dr. Bronsburg currently serves as a program committee member for the International Institute for Applied Knowledge Management Conference and is a peer reviewer for the American Medical Informatics Association Annual Symposium. 
Steven B. Zucker serves as Associate Dean for Community Affairs in the College of Osteopathic Medicine and Director of its Area Health Education Center (AHEC) Program. He is responsible for the College's community outreach programs, including his key role in the initial development and expansion of the College's highly respected Rural Medicine Program. Dr. Zucker received a D.M.D. from the University of Connecticut Health Sciences Center and was a Post-Doctorate Fellow in Dental Education at the University of Florida. He also holds a M.Ed. from the University of Hartford and a B.A. from the University of Pennsylvania. His professional career has spanned nearly forty years in medical and dental education, including prior service on the full-time Faculty of Eastern Virginia Medical School and Virginia Commonwealth University School of Dentistry. His success in acquiring significant extramural support has greatly assisted the College of Osteopathic Medicine in providing resources that are highly contributory to its clinical and academic curriculum. It has also served as a catalyst for the development of several other important NSUCOM community-based primary care and public health training initiatives in a wide-range of areas such as: tobacco prevention/cessation, minority health, HIV/AIDS, domestic and family violence, and cardiovascular disease prevention. 